\title{
Contents, Vol. 65, 1928
}

\section{Inhalts -Verzeichnis.}

Eigenarbeiten. 8eite

Berneaud, G., Traumatische maximale Pupillenenge . . 329 Birch-Hirschfeld, Neuere

Anschauungen über Trachom . 209

- Bemerkungen zur ,Lichttherapie des Auges” von

Urbanek $\quad 349$

Bothmann, L., s. Kronfeld, P.

Braunstein, N., Zur Frage der Behandlung von Augen-

erkrankungen mit ultravioletten Strahlen 232

Dinger, G.. Gefäße auf der vorderen Linsenkapsel in der

Pupille ohne merkliche Schwartenbildung .... 129

Elkes, G., Die keimfreien Bakterienfiltrate nach Besredkaund ihre therapeutische Anwendung

bei Augen-erkrankungen 135

Falta, M., Bemerkung zu der Arbeit: ,,Prüfung der Horn-hautempfindlichkeit

gegenDruckausübungam Lide als diagnostisches Hilfsmittel·' von Prof. Dr. W. Comberg 188

Filatow, W., Zur Technik der partiellen durchgreifenden

Keratoplastik 147

Ginzburg, J., Einiges über die sympathische Entzündung

und sympathische Reizung 24

Handmann, M. , Seröse Zyste der Orbita, von versprengter

Nasenschleimhaut ausgehend 321

Heygster, H., Pupillenstörungen und Lebensdauer . . 166

John, I.. Knötchenförmige Veränderung der Hornhaut-rückfläche bei Groenouw scher familiärer Hornhaut-dystrophie 240

Kranz, H. W., Kristallbildungen in der vorderen Augen-

kammer und Linse 219

Kronfeld, P. und Bothmann, L., Zur Frage der Linsenatmung 41

Mauksch, H., Über Versuche einer rhinologischen Behandlung der tabischen Optikusatrophie 336

Murad-Chan,Ali Bei, Membrana pupillaris persistenscorneaeadhaerens, eine Wimper in der vorderen Augen-kammer vortäuschend 346

Mussa-Beili, Uminissa Chanum Zur Kasuistik der ,,Chiffres

de Гlris" (van Duyse) 344

Mylius, K., Boecksches Sarkoid und Auge 71

Rabinowitsch, M., Über den Nystagmus monocularis . . 162

Reitsch, W., Der gesenkte Blick 244

- $\quad$ Lid- und Bulbusstellung beim geschlossenen Auge . 251Rutberg, M. N., Ein Fall von

Affektion der Tränenwege

durch Pilzkonkremente (Streptothrix)79 
Schindíer, E., Das Hämangioendotheliom des Oberlides und der Orbita im Säuglingsalter 150

Sommer, I., Labyrinth und Schielen 14

Siemens, H., Angeborene Beweglichkeitsstörungen der

Augenmuskeln

1

Vogelsang, K., Experimentelle Untersuchungen über die

Hornhautsensibilität mittels der Reaktionszeitmethode 63

$-\mathrm{IV}-$

S $\beta 1 \mathrm{t} \beta$

v. Volkmann, G., Über Fieberbehandlung verschiedenerAugenleiden mit einem neuen, unspezifischen Fieber-mittel (Pyrifer) 10

Zamkowski, J. G., Vergiítung mit Methylalkohol mit kon-

sekutiver Blindheit und Exitus letalis 237

Grüter, W., und Meyer, A., Zur Kasuistik wechselnder Pu-

pillenphänomene (Berichtigung) 82

Aus der Praxis fur die Praxis.

Carsten, P., Ein neuer Weg zur Bebandlung der Retinitis

pigmentosa?

189

Friede, R., Ein einfacher Handgriff zur Ausführung der

Druckspülung bei Verengerung der Tränenwege . . 83

Lose, P., Zur Herstellung provisoríscher Prothesen . . 256

Poljak, G. D., Synchisis scintillans camerae anterioris . 85

- Bemerkenswerter Fall eines Holzsplitters im Auge 353

Berichte über die deutsche ophthalmologische Literatur.

Augenmuskeln (1926) von Dozent Dr. E. Bachstez in Wien .... 258

Berichte über die ausländische ophthalmologische Literatur.

Bericht über die italienische Literatur (1925). Von Dr. C. Tirelli

in Bologna und Dr. E. Guzmann in Wien 88, 278

Gesellschaftsberichte.

Ophthalmologische Gesellschaftin Wien. Sitzung vom 23. April 1928191

Sitzung vom 21. Mai 1928294

Sitzung vom 18. Juni 1928356

Südostdeutsche Augenärztevereinig. 19 Tagung am 25726. Febr. 1928105

Berliner augenärztliche Gesellschaft. Sitzung vom 25. März 1928 . . 303

Bericht über die 52. Versammlung des Vereins Rheinisch-Westfäli-

scher Augenärzte (Jung-Stilling-Tagung) am Sonntag, den

25. März 1928 in Elberfeld 195

Verein der Augenärzte von Ost- und Westpreußen. 31. Sitzung am

26. November 1927 in Königsberg i. Pr. 305

Nordwestdeutsche augenärztliche Vereinigung. Sitzung vom 28. und

29. April im Ärztehaus zu Kannover $\quad 360$

Ungar. Ophthalmol. Ges. in Budapest. Sitzung vom 30. März 1928 375Vereinigte Med.

Gesellschaft in Kiew, Sektion für Augenheilkunde.

Sitzungen vom 18. Okt., 25. Okt. und 30. Nov. 1927 ....

Sitzungen vom 11. und 31. Januar $1928 \quad 309$

Diagnose und Therapie .... Unfall- und Versicherungskunde 
Blindenwesen

Buchbesprechungen

Tagesnachrichten

Personalien

Sachregister

Namenregister

$119,203,311,379 \cdot \cdots 123,312,383$

384

$125,208,317,385$

... 127, 320, 387

. 126, 320, 386

395 